\title{
THE NEAR CENTER OF AN ORTHOMODULAR LATTICE
}

\author{
M. F. JANOWITZ* \\ (Received 16 June 1970) \\ Communicated by P. D. Finch
}

\section{Introduction}

The results described herein were obtained in connection with the development of some lattice theoretic machinery needed by Randall and Foulis in their treatment of the so-called "logic of empirical science" (See [3] for an introduction to this subject). The final product, however, turned out to be of sufficient interest to warrant its independent presentation.

In the interest of brevity, our terminology and notation will, whenever possible, follow that of [1]. For that reason we shall not bother to re-introduce it here. Specifically, it is assumed that the reader is familiar with the material contained in [1], pp. 52-3. We shall make repeated use of the fact that every interval sublattice of an orthomodular lattice is itself an orthomodular lattice ([1], Corollary 3, p. 53).

Given the orthomodular lattice $L$, one says that $L$ is the disjoint sum of the family $\left(L_{\alpha}\right)_{\alpha \in A}$ and writes $L=D S\left(L_{\alpha}: \alpha \in A\right)$ in case there is an equivalence relation $E$ on $L \backslash\{0, I\}$ such that:

(1) $a C b \Rightarrow a E b$ (here $a C b$ denotes the fact that $a$ commutes with $b$ in the sense of [1], p. 52).

(2) The set of equivalence classes of $E$ is $\left\{L_{\alpha} \backslash\{0, I\}: \alpha \in A\right\}$.

(3) There exist $a, b \in L \backslash\{0, I\}$ such that $a E b$ fails. If $L=D S\left(L_{\alpha}: \alpha \in A\right)$ then each $L_{\alpha}$ is called a disjoint summand of $L$ and if there are only two summands $M$ and $N$, we shall simply write $L=D S(M, N)$ to denote the fact that $L$ is the disjoint sum of $M$ and $N$. It is important to notice that if $L=D S\left(L_{\alpha}: \alpha \in A\right)$, then each $L_{\alpha}$ may be regarded as an orthomodular sublattice of $L$. Given an indexed family $\left(L_{x}\right)_{\alpha \in A}$ of pairwise disjoint orthomodular lattices such that each $L_{\alpha}$ has at least four elements and Card $(A) \geqq 2$ one can easily form an orthomodular lattice $L$ such that

$$
L=D S\left(L_{\alpha}: \alpha \in A\right) \text {. }
$$

Letting $0_{\alpha}, I_{\alpha}$ denote the zero and unit elements of $L_{\alpha}$, we begin by setting

* Research supported by NSF Grant GP-11580. 


$$
M=\cup\left\{L_{\alpha} \backslash\left\{0_{\alpha}, I_{\alpha}\right\}: \alpha \in A\right\}
$$

and ordering $M$ by the rule $x \leqq y$ in $M \Leftrightarrow x \leqq y$ in some $L_{\alpha}$. Then let $L$ denote the lattice formed by adjoining a new zero and unit element to $M$. If one uses the induced orthocomplementation on $L$, the result is the desired orthomodular lattice.

As a final introductory item, it will prove convenient to agree at this point that $L$ shall always denote an orthomodular lattice throughout the remainder of this paper.

\section{The near center}

An element $e$ of an orthomodular lattice $L$ is called upper nearly central if $e \leqq a<I$ implies $e$ central in $[0, a]$; it is called lower nearly central if $0<b \leqq e$ implies $e$ central in $[b, I]$ and nearly central if it is both upper and lower nearly central. Evidently $e$ is upper nearly central $\Leftrightarrow e^{\perp}$ is lower nearly central. Examples of upper nearly central elements are provided by: (1) any central element; (2) any central element of a disjoint summand of $L$; (3) any co-atom of $L$ (i.e., any element covered by $I$ ). The first two examples are of course nearly central, and we shall soon prove that every nearly central element arises in this manner. Further examples of upper nearly central elements may be constructed by judicious application of the next lemma.

LEMMA 1. Let $f \leqq e$ in L. If $e$ is upper nearly central in $[f, I]$ and $f$ is upper nearly central in $L$, then $e$ is upper nearly central in $L$.

Proof. Given $e \leqq a<I$ we have $f \leqq e \leqq a<I$, so $e$ is central in $[f, a]$ and $f$ is central in $[0, a]$. It is immediate that $e$ is central in $[0, a]$.

We now develop a few elementary properties of upper nearly central elements. In connection with this it will prove convenient to write $e \sim f$ in case $e$ and $f$ have a common complement in $L$ (i.e., in case $e$ is perspective to $f$ ).

LEMMA 2. Let $e, f$ be upper nearly central. If e does not commute with $f$ then $e \sim f$.

Proof. By definition of upper nearly central we must have

$$
e \vee f=e \vee f^{\perp}=e^{\perp} \vee f=I
$$

so $e \vee f^{\perp}=I$ and $e \wedge f^{\perp}=0$. Thus $f^{\perp}$ is a common complement for $e$ and $f$.

THEOREM 3. Let $e$ be upper nearly central but not central. If $e<a<I$ then $e \sim a$.

Proof. If $e C x$ for all complements $x$ of $a$, then $e \wedge x=0$ forces $e \leqq x^{\downarrow}$. Thus $e$ is contained in all complements of $a^{\perp}$. By [1], Lemma 2, p. $71 e \vee y=I$ 
implies $a^{\perp} \leqq y$. But then $e, y^{\perp} \in[0, a]$ forces $e C y^{\perp}$ and consequently $e C y$. Since $e \wedge y<I$ also implies $e C y$ we see that $e$ is central, a contradiction. It follows that there must exist a complement $x$ of $a$ such that $e$ does not commute with $x$. Then $e \vee x=I$ and since $e \leqq a$, we must have $e \wedge x=0$. Thus $x$ is a common complement for $e$ and $a$.

COROLLARY. If $L$ is modular, then every upper nearly central element of $L$ is a co-atom.

We mention here that it is a fairly easy matter to extend the above Corollary to the case where $L$ has the property that $e$ central in $[0, a]$ implies that $e$ is contained in all complements of $a \wedge e^{\perp}$. The following innocuous appearing lemma will turn out to have some important and far-reaching consequences (See, for example, the proof of Lemma 17).

LEMMA 4. If e is upper nearly central but not central then for each central element $z$ either $z \leqq e$ or $z^{\perp} \leqq e$.

Proof. Since $e$ is not central there must exist an $x \in L$ such that $e C x$ fails. Since

$$
x=(x \wedge z) \vee\left(x \wedge z^{\perp}\right),
$$

it follows from [1], Theorem 22, p. 53 that $x \wedge z$ or $x \wedge z^{\perp}$ must fail to commute with $e$. If $x \wedge z$ does not commute with $e$, then

$$
I=e \vee(x \wedge z) \Rightarrow e \vee z=I,
$$

so $e \geqq z^{\perp}$. Similarly, $x \wedge z^{\perp}$ not commuting with $e$ forces $e \geqq z$.

LEMMA 5. If $e$ is upper nearly central and $x \neq e^{\perp}$ is a complement of $e$ then $0<y \leqq x$ implies $y \notin C(e)$.

Proof. Since $x \neq e^{\perp}$, we cannot have $x C e$. It follows that $e \vee x^{\perp}=I$ so $x \wedge e^{\perp}=0$. If $0<y \leqq x$ and $y C e$ then $y \wedge e=0$ would force $y \leqq e^{\perp}$, so $y \leqq e^{\perp} \wedge x=0$, a contradiction. Thus $0<y \leqq x$ implies $y \notin C(e)$.

We are now ready to consider the nearly central elements of $L$. To this end, we agree to let $N C(L)$ denote the set of nearly central elements of $L$, and call $N C(L)$ the near center of $L$, the symbol $C(L)$ being reserved for the center of $L$.

THEOREM 6. Let $e \perp f$ and assume that $e, f$ are both upper nearly central elements of $L$, neither of which is central. Then $C(e)$ is a disjoint summand of $L$.

Proof. (a) We begin by showing that $C(e)=C(f)$. To see this, observe that $x \notin C(e)$ implies $x^{\perp} \vee e=I$ so that $x \wedge e^{\perp}=0$. If $x C f$ we would have $x \leqq f^{\perp}$ since $x \wedge f=0$. But then $e \vee x \leqq f^{\perp}<I$ would imply $e C x$, a contradiction. Thus 


$$
L \backslash C(e) \subseteq L \backslash C(f),
$$

and a symmetric argument produces the reverse inclusion.

(b) We next show that $x \notin C(e)$ and $0<y \leqq x$ together imply that $y \notin C(e)$. In view of Lemma 5 , we need only show that $x \notin C(e) \Rightarrow x$ is a complement of $e$. Since $x \vee e=I$ is automatic, we merely observe here that

$$
0 \leqq x \wedge e=\left(x^{\perp} \vee e^{\perp}\right)^{\perp} \leqq\left(x^{\perp} \vee f\right)^{\perp}=I^{\perp}=0
$$

since by (a), $x \notin C(e) \Rightarrow x \notin C(f)$.

(c) Finally, we define an equivalence relation $E$ on $L \backslash\{0, I\}$ by the rule

$$
x E y \text { if either }\{x, y\} \subseteq C(e) \text { or }\{x, y\} \subseteq L \backslash C(e) .
$$

In order to show that $C(e)$ is a disjoint summand of $L$, the only nontrivial assertion is that $x C y \Rightarrow x E y$. This would follow if we could just establish that

$$
x \notin C(e), x C y \Rightarrow y \notin C(e) .
$$

There are two possible cases: Case 1. $x \wedge y \neq 0$. Then by (b), $x \wedge y \notin C(e)$ so $x^{\perp} \vee y^{\perp} \notin C(e), y^{\perp} \notin C(e)$ and consequently $y \notin C(e)$. Case 2. $x \wedge y=0$. Since $x C y$ this forces $y \leqq x^{\perp}$, and by (b), $y \notin C(e)$.

As an immediate consequence of the above theorem we have the following characterization of the near center of $L$.

THEOREM 7. Given $e \in L$, the following conditions are equivalent:

(1) e is nearly central but not central.

(2) Both e and $e^{\perp}$ are upper nearly central, but $e$ is not central.

(3) $e$ is upper nearly central but not central and there exists an element $f$ of $L$ such that $e \perp f, f$ is upper nearly central, and $f$ is not central.

(4) $e$ is a central element of a disjoint summand of $L$.

At this point we need to state

Lemma 8. Let e, $f$ be nearly central elements of $L$. Then $C(e)=C(f)$ or $C(e) \cap C(f)=\{0, I\}$.

Proof. If $C(e) \neq C(f)$ we may clearly assume the existence of an element $x$ such that $x C f$ but $x \notin C(e)$. Then $e$ is not central so by Theorem 6 ,

$$
L=D S(C(e), N)
$$

where $N=[L \backslash C(e)] \cup\{0, I\}$. Since $0<x<I$ and $x \in C(f) \cap N$ we must have $C(f) \subseteq N$ so $C(e) \cap C(f)=\{0, I\}$.

This now allows us to prove the following fundamental theorem about the near center of $L$. 
THEOREM 9. Let $L$ be an orthomodular lattice.

(1) If there exists a central element $z \notin\{0, I\}$ then $N C(L)=C(L)$.

(2) If there exists a nearly central element which is not central, then every nearly central element is a central element of a disjoint summand of L. It follows that $N C(L)$ is a sublattice of $L$, and as such is either a Boolean algebra or a disjoint sum of Boolean algebras.

Proof. (a) If there exists a central element $z \notin\{0, I\}$ then $L$ can admit no disjoint sum decomposition. In view of Theorem 7. We must have $C(L)=N C(L)$.

(b) Assume now that there exists a nearly central element which is not central. Then by (a), $C(L)=\{0, I\}$. It follows from Theorem 7 that every nearly central element is a central element of a disjoint summand of $L$. If the nearly central elements all commute, then they must all lie in the same disjoint summand. In this case $N C(L)$ is a Boolean sublattice of $L$. Suppose on the other hand that there exist nearly central elements $z_{1}$ and $z_{2}$ which do not commute. Let

$$
M=\cup\{C(z): 0<z<I, z \in N C(L)\} .
$$

If $M \neq L$, it follows quickly from Theorem 7 that $M$ is a disjoint summand of $L$. In order to prove the remaining assertion of the theorem, we may clearly restrict our attention to $M$. Accordingly, we define an equivalence relation $E$ on $M \backslash\{0, I\}$ by the rule

$x E y$ if and only if $\{x, y\} \in C(z)$ for some $z$ in $N C(L) \backslash\{0, I\}$.

It follows from Lemma 8 that $x C y \Rightarrow x E y$ so $E$ induces a disjoint sum decomposition

$$
M=D S\left(L_{\alpha}: \alpha \in A\right) .
$$

Now $x \in L_{\alpha}$ implies $x E x$ so $x C z$ for some $z \in N C(L) \backslash\{0, I\}$. Clearly $C(z) \subseteq L_{z}$ and if $y \in L_{x} \backslash\{0, I\}$, then $\{y, z\} \subseteq C(w)$ for some $w \in N C(L) \backslash\{0, I\}$. By Lemma 8 $C(z)=C(w)$, so $y C z$. It follows that $L_{\alpha}=C(z)$ and that

$$
N C(L) \cap L_{x}=C\left(L_{x}\right) \text {. }
$$

It is immediate that $N C(L)$ is a sublattice of $L$ and that the restriction of $E$ to $N C(L)$ induces a disjoint sum decomposition

$$
N C(L)=D S\left(N_{\alpha}: \alpha \in A\right)
$$

where $N_{\alpha}=C\left(L_{\alpha}\right)$ for all $\alpha \in A$.

\section{Indexing chains}

A finite chain of the form

$$
I=a_{0}>a_{1}>\cdots>a_{n}=a
$$


where $a_{i}$ is a nearly central element of $\left[0, a_{i-1}\right]$ for $i=1,2, \cdots, n$ and $a_{n}>0$ is called an indexing chain connecting $a$ with $\mathrm{I}$ and the non zero element $a$ is called indexed if such a chain exists. We agree to call the orthomodular lattice $L$ indexed if every element of $L$ is the join of a family of indexed elements. Our reasons for looking at this concept are two-fold: First of all, it turns out that this is the type of lattice encountered by Randall and Foulis in their investigation of empirical logic; secondly, this seemingly unrelated concept sheds considerable light on the nature of upper nearly central elements.

Let us say that the indexing chain

$$
I=a_{0}>a_{1}>\cdots>a_{n}
$$

has length $n$, and that it is minimal if for $i=1, \cdots, n-1$ the chain

$$
I=a_{0}>\cdots>a_{i-1}>a_{i+1}>\cdots>a_{n}
$$

formed by deleting $a_{i}$ is not an indexing chain. Evidently any indexed element may be connected with $I$ by at least one minimal indexing chain. Our first order of business will be to show that the existence of minimal indexing chains of length 2 forces $N C(L)$ to have atoms. This fact will then be used to prove that every indexed element of an orthomodular lattice has a unique minimal indexing chain connecting it with $I$.

LEMMA 10. The indexing chain

$$
I=a_{0}>a_{1}>a_{2}
$$

is minimal if and only if $a_{1}$ is an atom of $N C(L)$.

Proof. If the given chain is not minimal, then $I=a_{0}>a_{2}$ must be an indexing chain. But now $a_{2} \in N C(L)$ with $a_{2}<a_{1}$ shows $a_{1}$ is not an atom of $N C(L)$. Suppose on the other hand that $a_{1}$ is not an atom of $N C(L)$. Then there exists a nearly central element $z$ with $0<z<a_{1}$. We now observe that $z$ is central in $\left[0, a_{1}\right]$ and it follows from Theorem 9 that $a_{2}$ is central in $\left[0, a_{1}\right]$. If $a_{1}$ is central in $L$, then $a_{2}$ central in $\left[0, a_{1}\right]$ forces $a_{2}$ central in $L$; similarly, $a_{1}$ central in a disjoint summand of $L$ forces $a_{2}$ central in that summand. In either event $a_{2} \in N C(L)$, so $I=a_{0}>a_{2}$ is an indexing chain, and the original chain was not minimal.

THEOREM 11. An indexing chain

$$
I=a_{0}>a_{1}>\cdots>a_{n}>a_{n+1}
$$

is minimal if and only if $a_{i}$ is an atom of $N C\left(\left[0, a_{i-1}\right]\right)$ for $i=1,2, \cdots, n$.

Proof. If the condition holds then by Lemma 10, no $a_{i}$ may be removed $(1 \leqq i \leqq n)$. Hence the chain is minimal. If the condition fails, then some $a_{i}$ 
$(1 \leqq i \leqq n)$ is not an atom of $N C\left(\left[0, a_{i-1}\right)\right]$. Apply Lemma 10 to the indexing chain

$$
a_{i-1}>a_{i}>a_{i+1}
$$

of $\left[0, a_{i-1}\right]$ to get that $a_{i-1}>a_{i+1}$ is an indexing chain in that interval. It is immediate that

$$
I=a_{0}>a_{1}>\cdots>a_{i-1}>a_{i+1}>\cdots>a_{n}>a_{n+1}
$$

is an indexing chain in $L$, so that the original chain was not minimal.

THEOREM 12. Let a be an indexed element of $L$. There then exists a unique minimal indexing chain connecting a with $I$.

Proof. The existence of at least one such chain is clear, so we shall direct our attention toward establishing uniqueness. We will proceed by induction on the length of a minimal indexing chain. We begin with the case where there is a minimal indexing chain of length 1 . If

$$
I=b_{0}>b_{1}>\cdots>b_{n}=a
$$

is any other indexing chain, then so is $I=b_{0}>b_{n}=a$ so we have a unique minimal indexing chain connecting $a$ with $I$. Suppose now that the theorem is true for all indexed elements of an orthomodular lattice having a minimal indexing chain of length at most $n$. Let

$$
I=a_{0}>a_{1}>\cdots>a_{n}>a_{n+1}=a
$$

be a minimal indexing chain of length $n+1$ and let

$$
I=b_{0}>b_{1}>\cdots>b_{k}=a
$$

denote any other minimal indexing chain, where we may clearly assume that $1 \leqq n<k$. Then

$$
a_{1} \wedge b_{1} \geqq a>0,
$$

and by Theorem 11, $a_{1}, b_{1}$ are atoms of $N C(L)$. It follows that $a_{1}=b_{1}$. Working in the orthomodular lattice $\left[0, a_{1}\right]$ we see that

$$
a_{1}>\cdots>a_{n}>a_{n+1}=a
$$

is a minimal indexing chain of length $n$. By our induction hypothesis we see that $k=n+1$ and $a_{i}=b_{i}$ for all $i$.

Before proceeding, we shall have a look at modularity and commutativity of indexed elements.

THEOREM 13. Let $a, b$ denote indexed elements of L. If $a \wedge b \neq 0$ then $a \wedge b, a \vee b$ are indexed and $a C b$ 
Proof. Let

$$
\begin{aligned}
& I=a_{0}>a_{1}>\cdots>a_{k}>a_{k+1}=a \\
& I=b_{0}>b_{1}>\cdots>b_{n}>b_{n+1}=b
\end{aligned}
$$

denote minimal indexing chains. We may clearly assume $k \leqq n$. If $k \geqq 1$, then

$$
a_{1} \wedge b_{1} \geqq a \wedge b>0
$$

with $a_{1}, b_{1}$ atoms of $N C(L)$ implies $a_{1}=b_{1}$. Similarly, $a_{i}=b_{i}$ for $i=2, \cdots, k$. By dropping down to the interval $\left[0, a_{k}\right]$ we may assume that $a$ is nearly central in $L$. If $b$ is nearly central there is nothing to prove, and otherwise,

$$
a \wedge b_{1} \geqq a \wedge b>0
$$

with $b_{1}$ an atom of $N C(L)$ forces $b_{1} \leqq a$. But now $b \leqq a$, and once again there is nothing to prove.

In connection with the next theorem, we shall use the symbol $M(a, b)$ to denote the fact that $(a, b)$ forms a modular pair in the sense that

for all $c \in L$.

$$
c \leqq b \Rightarrow(c \vee a) \wedge b=c \vee(a \wedge b)
$$

THEOREM 14. Let $a, b$ denote indexed elements of $L$ with $a \wedge b=0$. Then $M(a, b) \Leftrightarrow b$ is an atom or $a \perp b$.

Proof. It suffices to prove that if $b$ is not an atom, then $M(a, b) \Rightarrow a \perp b$. Let

$$
\begin{aligned}
& I=a_{0}>a_{1}>\cdots>a_{k}>a_{k+1}=a \\
& I=b_{0}>b_{1}>\cdots>b_{n}>b_{n+1}=b
\end{aligned}
$$

denote minimal indexing chains. We break the proof into three cases.

Case $1 . n \leqq k$ and $a_{i}=b_{i}$ for $i \leqq n$. Dropping to $\left[0, a_{n}\right]$ we may as well assume that $b$ is nearly central in $L$ with $a$ indexed. If $b$ is central there is nothing to prove; otherwise, we may write $L=D S(M, N)$ with $b$ central in $M$. If $a \notin M$, we choose an element $c$ such that $0<c<b$. Then

$$
c=c \vee(a \wedge b)<(c \vee a) \wedge b=b,
$$

contrary to $M(a, b)$. Thus $a \in M$ so $a C b$ and therefore $a \perp b$.

Case 2. $k \leqq n$ and $a_{i}=b_{i}$ for $i \leqq k$. Here we drop down to $\left[0, a_{k}\right]$ so that we may assume $a$ nearly central in $L$ with $b$ indexed. The proof is now analogous to Case 1 .

Case 3. $a_{i} \neq b_{i}$ for some $i \leqq \min (k, n)$. Then both $k$ and $n$ are greater than 0 , and we may take $i$ to be the least positive integer with the indicated property. 
Thus $a_{i-1}=b_{i-1}$ and by dropping down to $\left[0, a_{i-1}\right]$ we may as well assume that $a_{1} \neq b_{1}$. If $a_{1} \notin C\left(b_{1}\right)$ we may write $L=D S(M, N)$ with $a_{1} \in M \backslash\{0, I\}$ and $b_{1} \in N \backslash\{0, I\}$. Then $a \in M, b \in N$ and as in Case $1, M(a, b)$ fails. Thus we must have $a_{1} C b_{1}$, and since $a_{1}, b_{1}$ are atoms of $N C(L), a_{1} \perp b_{1}$ follows. Therefore, $a \perp b$ as desired.

THEOREM 15. Let $L$ be an indexed orthomodular lattice having no atoms. Then $M(a, b) \Leftrightarrow a C b$.

Proof. That $a C b \Rightarrow M(a, b)$ follows from [1], Theorem 23, p. 53. To obtain the converse we use the fact that the mapping

$$
x \rightarrow x \wedge\left(a^{\perp} \vee b^{\perp}\right)
$$

is an isomorphism of the interval $[a \wedge b, a \vee b]$ onto the interval

$$
\left[0,(a \vee b) \wedge\left(a^{\perp} \vee b^{\perp}\right)\right] \text {. }
$$

Then $M(a, b)$ implies $M\left(a \wedge\left(a^{\perp} \vee b^{\perp}\right), b \wedge\left(a^{\perp} \vee b^{\perp}\right)\right)$ and

$$
\left[a \wedge\left(a^{\perp} \vee b^{\perp}\right)\right] \wedge\left[b \wedge\left(a^{\perp} \vee b^{\perp}\right)\right]=0 .
$$

If $a_{1} \leqq a \wedge\left(a^{\perp} \vee b^{\perp}\right), b_{1} \leqq b \wedge\left(a^{\perp} \vee b^{\perp}\right)$ are indexed, then by Theorem 14, $M\left(a_{1}, b_{1}\right)$ implies $a_{1} \perp b_{1}$. Since $L$ is indexed

$$
a \wedge\left(a^{\perp} \vee b^{\perp}\right) \perp b \wedge\left(a^{\perp} \vee b^{\perp}\right)
$$

follows. By [2], Lemma 3, p. 68 this yields $a C b$.

As a final item we now seek to relate the notion of an indexed element with that of an upper nearly central element. We begin with the observation that by repeated application of Lemma 1, we have that every indexed element of an orthomodular lattice is lower nearly central. As a partial converse to this we present

LEMMA 16. Let e be an upper nearly central element of $L$. If $e^{\perp}$ contains an indexed element then $e^{\perp}$ is itself indexed.

Proof. We may clearly assume that $e$ is not nearly central since in that case there is nothing to prove. We will proceed by induction on the length of a minimal indexing chain connecting an indexed subelement of $e^{\perp}$ with $I$, the length 1 case being trivial by Theorem 7 .

Suppose that there exists a minimal indexing chain

$$
I=a_{0}>a_{1}>\cdots>a_{n}
$$

with $a_{n} \leqq e^{\perp}$. If $a_{1}$ is central then by Lemma 4 , either $a_{1} \leqq e$ or $a_{1}^{\perp} \leqq e$. If $a_{1}$ is not central then $C\left(a_{1}\right)$ is a disjoint summand of $L$. Now

$$
e^{\perp} \wedge a_{1} \neq 0 \Rightarrow e C a_{1}
$$


and since $e \notin N C(L)$ we must have that $e$ is upper nearly central but not central in $C\left(a_{1}\right)$. Applying Lemma 4 to $C\left(a_{1}\right)$, we once more obtain $a_{1} \leqq e$ or $a_{1}{ }^{\perp} \leqq e$. Now if $a_{1} \leqq e$ then $a_{n} \leqq a_{1} \leqq e$ forces

$$
a_{n} \leqq e \wedge e^{\perp}=0,
$$

contrary to $a_{n}>0$. We deduce that $a_{1}^{\perp} \leqq e$, so $e^{\perp} \leqq a_{1}$.

We claim next that $e \wedge a_{1}$ is upper nearly central in $\left[0, a_{1}\right]$. To see this, let

$$
e \wedge a_{1} \leqq x<a_{1}
$$

Then

$$
\left(e \wedge a_{1}\right) \vee a_{1}{ }^{\perp}=e \vee a_{1}{ }^{\perp} \leqq x \vee a_{1}{ }^{\perp}<I .
$$

If $y \leqq x$, then $e, y \in\left[0, x \vee a_{1}{ }^{\perp}\right]$ forces $e C y$, and consequently $e \wedge a_{1} C y$. It follows that $e \wedge a_{1}$ is central in $[0, x]$, so $e \wedge a_{1}$ is upper nearly central in $\left[0, a_{1}\right]$. If it were nearly central this would make $e^{\perp}$ nearly central in $\left[0, a_{1}\right]$ thus completing the proof; if not, we drop down to $\left[0, a_{1}\right]$ and note that

$$
a_{1}>a_{2}>\cdots>a_{n}
$$

is a minimal indexing chain of length $n-1$. By induction we may assume $e^{\perp}$ indexed in $\left[0, a_{1}\right]$ and consequently in $L$.

This leads immediately to

THEOREM 17. An element $e \notin\{0, I\}$ of an indexed orthomodular lattice $L$ is upper nearly central $\Leftrightarrow e^{\perp}$ is indexed; it is nearly central $\Leftrightarrow$ both $e$ and $e^{\perp}$ are indexed.

Thus Theorems 13 and 14 may be dualized to apply to upper nearly central elements. We summarize the results here: Let $a, b$ denote upper nearly central elements of the indexed orthomodular lattice $L$.

(1) If $a \bigvee b<I$, then $a \wedge b, a \bigvee b$ are upper nearly central.

(2) If $a \bigvee b=I$ then $M^{*}(a, b) \Leftrightarrow b$ is a co-atom or $a \geqq b^{\perp}$.

\section{References}

[1] G. Birkhoff, Lattice theory (Amer. Math. Soc. Colloq. Publ. XXV, 3rd Edition, Providence, R. I., 1967).

[2] D. J. Foulis, 'Relative inverses in Baer *-semigroups', Michigan Math. J. 10 (1963), 65-84.

[3] C. H. Randall and D. J. Foulis, 'An approach to empirical logic', Amer. Math. Monthly 77 (1970), 363-374.

University of Massachusetts

Amherst, Massachusetts

U.S.A. 